\title{
Editorial
}

\section{Carbon Nanomaterials and Related Nanostructures: Synthesis, Characterization, and Application}

\author{
Jinlong Jiang, ${ }^{1}$ Jun Yang, ${ }^{2}$ Jian Lin, ${ }^{3}$ Zhenghong Huang, ${ }^{4}$ and S. C. Wang ${ }^{5}$ \\ ${ }^{1}$ Department of Physics, Lanzhou University of Technology, Lanzhou 730050, China \\ ${ }^{2}$ State Key Laboratory of Solid Lubrication, Lanzhou Institute of Chemical Physics, Chinese Academy of Sciences, \\ Lanzhou 730000, China \\ ${ }^{3}$ Department of Mechanical Engineering \& Materials Science, Rice University, Houston, TX 77005, USA \\ ${ }^{4}$ School of Materials Science and Engineering, Tsinghua University, Beijing 100084, China \\ ${ }^{5}$ National Centre for Advanced Tribology at Southampton, University of Southampton, Southampton SO17 1BJ, UK \\ Correspondence should be addressed to Jinlong Jiang; jinlong@lut.cn
}

Received 24 July 2014; Accepted 24 July 2014; Published 31 August 2014

Copyright (C) 2014 Jinlong Jiang et al. This is an open access article distributed under the Creative Commons Attribution License, which permits unrestricted use, distribution, and reproduction in any medium, provided the original work is properly cited.

\begin{abstract}
Since the discovery of buckyballs in 1985 , the family of carbon materials has been rapidly growing. From zero-dimensional (0D) fullerene, $1 \mathrm{D}$ carbon nanotubes (CNTs), and recent $2 \mathrm{D}$ graphene to traditional 3D nanoporous carbon and nanodiamond, carbon nanomaterials exhibit a variety of structures and forms due to the existence of three hybridization forms, namely, $\mathrm{sp}^{3}, \mathrm{sp}^{2}$, and $\mathrm{sp}^{1}$, of carbon atoms.

In recent years, they have increasingly gained interests from both scientific and industrial communities due to the unique physical and chemical properties. They show diverse potential applications in structure, electronics, energy, environment, water, and others. The purpose of this special issue is to report some latest research results on synthesis, characterization, and applications of carbon nanomaterials and related nanostructures. The accepted papers cover various carbon materials, namely, CNTs, graphene, carbon nanofibers, nanodiamonds, amorphous carbon films, carbon nanocomposite, graphite-like layered materials, and so on. These papers cover a wide range of topics related to synthesis and applications of these carbon nanomaterials in mechanics and tribology, thermal management, energy conversion (photoelectronic devices, solar cells, and photocatalysis), energy storage (lithium-ion batteries), and drug delivery fields. Moreover, two papers present theoretical calculation of carbon nanomaterials in electronic, optical, and surface adsorption properties using density functional theory.
\end{abstract}

The guest editors hope that this special issue will be of great interest to readers.

\section{Acknowledgments}

We would like to thank all the authors for their contributions to this special issue as well as the reviewers for their constructive comments to the manuscripts. We also want to express our sincere thanks to the editorial board members of Journal of Nanomaterials for the support and help in preparation of this special issue.

Jinlong Jiang Jun Yang Jian Lin Zhenghong Huang S. C. Wang 

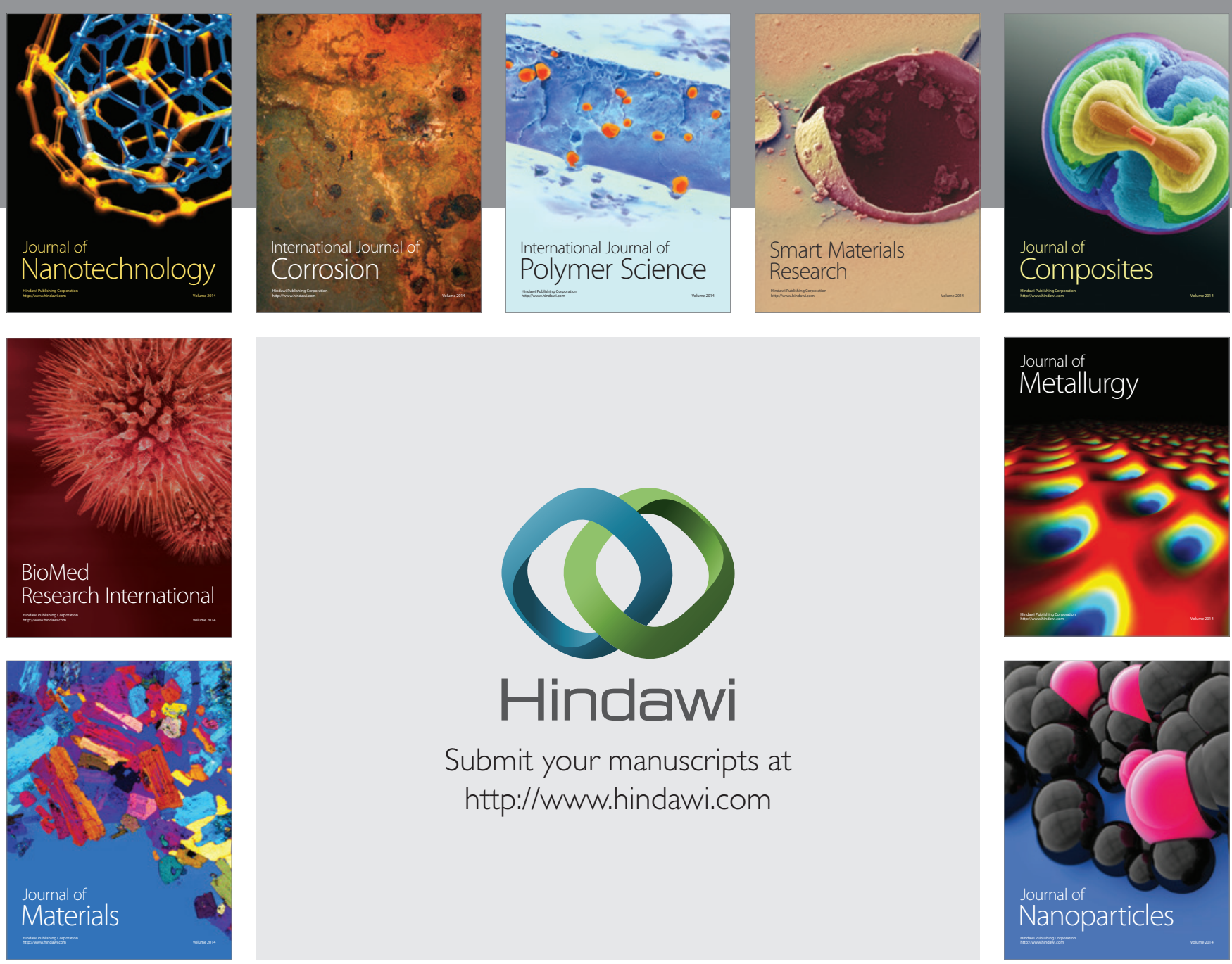

Submit your manuscripts at http://www.hindawi.com
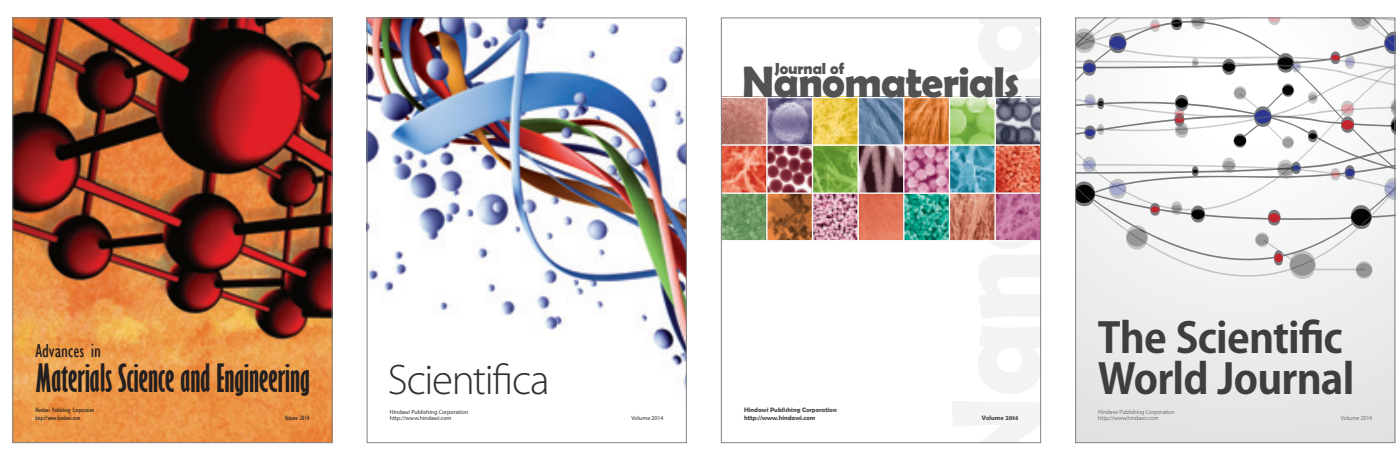

\section{The Scientific World Journal}
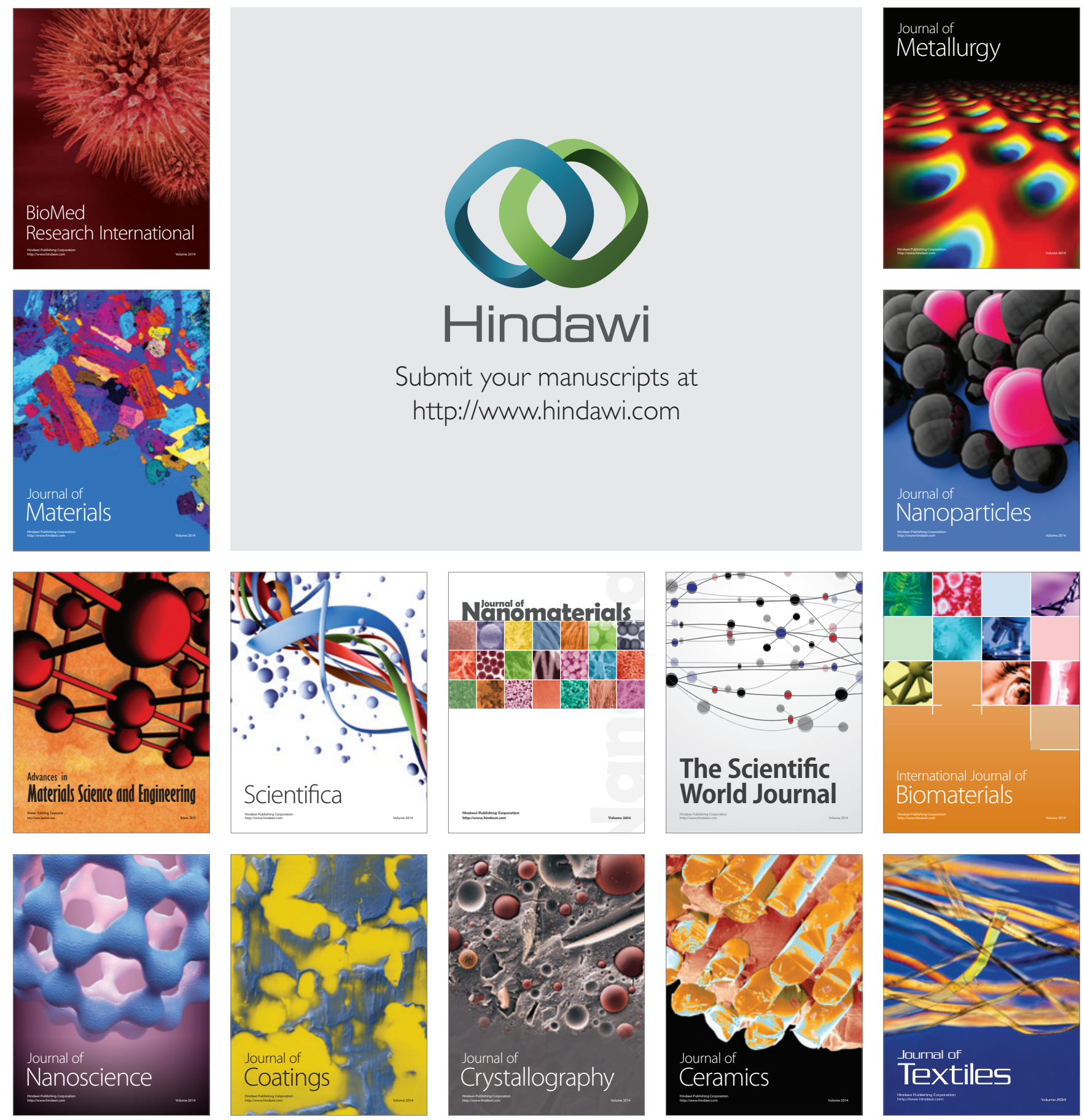